\title{
Abundancia estacional y distribución vertical del zooplancton gelatinoso carnívoro en una área de surgencia en el norte del Sistema de la Corriente de Humboldt
}

\author{
Sergio Palma \& Pedro Apablaza \\ Escuela de Ciencias del Mar, Pontificia Universidad Católica de Valparaíso \\ Casilla 1020, Valparaíso, Chile \\ e-mail: spalma@ucv.cl
}

\begin{abstract}
RESUMEN. Se analizó la abundancia estacional y distribución vertical del zooplancton gelatinoso colectado en cuatro cruceros efectuados frente a la bahía de Mejillones. En esta zona se identificaron 44 especies macroplanctónicas distribuidas en 23 de sifonóforos, 17 de hidromedusas, y 4 de ctenóforos, de las cuales 8 especies, Sphaeronectes irregularis, Rhizophysa eysenhardti, Amphogona apicata, Proboscidactyla stellata, Sarsia coccometra, Pleurobrachia bachei, Thalassocalyce inconstans y Velamen parallelum, se registran por primera vez para el Sistema de la Corriente de Humboldt. Se determinó un incremento de abundancia en primavera y verano, con densas agregaciones $(>2.600$ ind $100 \mathrm{~m}^{-3}$ ) en octubre de Aglaura hemistoma, Solmundella bitentaculata, Muggiaea atlantica y Pleurobrachia bachei, y en febrero de Obelia spp. Las mayores densidades se determinaron en la estación más costera y en el frente oceánico, mientras que en la estación oceánica se registró una fuerte disminución de abundancia. La mayoría de las especies dominantes se encontraron en los primeros $100 \mathrm{~m}$ de la columna de agua, con una mayor concentración de ejemplares en los primeros 25 o $50 \mathrm{~m}$ de profundidad. Esta distribución vertical estaría limitada por la capa de mínimo oxígeno $(<$ $\left.1 \mathrm{ml} \cdot \mathrm{L}^{-1}\right)$ que se detectó en aguas muy someras $(30-50 \mathrm{~m})$ durante los eventos de surgencia.
\end{abstract}

Palabras claves: zooplancton gelatinoso, abundancia estacional, distribución vertical, bahía de Mejillones, Chile.

\section{Seasonal abundance and vertical distribution of the carnivorous gelatinous zooplankton in an upwelling area of the northern Humboldt Current System}

\begin{abstract}
The seasonal abundance and vertical distribution of gelatinous zooplankton collected during four cruises off Mejillones bay were analyzed. In this area we identified 44 species of macroplankton distributed in 23 siphonophores, 17 hydromedusae, and 4 ctenophores. Eight species, Sphaeronectes irregularis, Rhizophysa eysenhardti, Amphogona apicata, Proboscidactyla stellata, Sarsia coccometra, Pleurobrachia bachei, Thalassocalyce inconstans and Velamen parallelum, are the first records in the Humboldt Current System. The seasonal distribution showed an increase in abundance in spring and summer, with the highest aggregations (>2600 ind $100 \mathrm{~m}^{-3}$ ) of Aglaura hemistoma, Solmundella bitentaculata, Muggiaea atlantica and Pleurobrachia bachei in October and Obelia spp. in February. The highest densities were observed in the coastal station and in the oceanic front, while the oceanic station was characterized by a strong decrease in abundance. The vertical distribution showed that the most dominant species were founded in the first $100 \mathrm{~m}$ of the water column, with a higher concentration of organisms in the first 25 or $50 \mathrm{~m}$ of depth. This vertical distribution could be limited by presence of a minimum dissolved oxygen layer $\left(<1 \mathrm{ml} \cdot \mathrm{L}^{-1}\right)$ in shallow waters $(30-50$ $\mathrm{m}$ ) during de upwelling events.
\end{abstract}

Key words: gelatinous zooplankton, seasonal abundance, vertical distribution, Mejillones Bay, Chile. 


\section{INTRODUCCIÓN}

En los últimos años se han incrementado los estudios orientados a comprender la distribución espacio-temporal de los organismos gelatinosos carnívoros y su rol ecológico en los sistemas costeros, debido a su elevado impacto trófico. Las medusas, sifonóforos y ctenóforos son predadores que actúan en los primeros niveles de la cadena trófica en el ecosistema marino, afectando la abundancia de estadios larvarios y adultos de diversos organismos zooplanctónicos, especialmente copépodos, y en ocasiones compiten con los peces pelágicos por el alimento (Alvariño, 1985; Purcell, 1985, 1991, 1997; Fancett \& Jenkins, 1988; Matsakis \& Conover, 1991; Pagès et al., 2001).

Los estudios sobre el zooplancton gelatinoso del Pacífico suroriental son escasos y se han realizado principalmente frente a la costa norte y central de Chile. En esta zona, se registra una escasa abundancia y diversidad específica, a excepción de los períodos cálidos de primavera y verano, cuando en zonas costeras aparecen densas agregaciones de carnívoros gelatinosos, que proliferan rápidamente, como Aglaura hemistoma, Liriope tetraphylla, Clytia spp., Obelia spp., Muggiaea atlantica, Sphaeronectes gracilis, Bassia bassensis y Thalassocalyce inconstans (Palma, 1977, 1994; Palma \& Rosales, 1995; Pagès et al., 2001).

La distribución temporal de los organismos gelatinosos en zonas costeras está fuertemente influenciada por gradientes de temperatura, que pueden afectar positivamente la abundancia y diversidad de sus poblaciones (Palma \& Rosales, 1995; Gasca, 1996; Marazzo \& Nogueira, 1996). Los incrementos de abundancia se registran preferentemente en el período estival y son comunes en áreas de surgencia costera de los márgenes orientales, donde el aumento de la productividad biológica (Morales et al., 1996), favorece el desarrollo de sus presas potenciales (Pagès \& Gili, 1992; Hutchings et al., 1995).

La península de Mejillones, ubicada en el norte de Chile, posee una estrecha plataforma continental que está afectada permanentemente por eventos de surgencia que ocurren en una angosta franja costera. La intensidad de estos eventos es mayor en primavera y causa altas tasas de producción primaria, con una media de $3.170 \mathrm{mgC} \cdot \mathrm{m}^{-2} \cdot \mathrm{d}^{-1}$ en la zona costera (González et al., 1998). La alta frecuencia e intensidad de estos eventos sustenta densas poblaciones de zooplancton quitinoso, caracterizadas por la dominancia de Calanus chilensis y Euphausia mucronata (Escribano et al., 2000). Frente a la península se han determinado diversas especies gelatinosas, algunas formando grandes agregaciones como el sifonóforo Bassia bassensis, que puede causar un alto impacto trófico sobre poblaciones de copépodos y puede remover hasta un $17 \%$ de su biomasa (Pagès et al., 2001).

Las aguas de la zona norte pertenecen al Sistema de la Corriente de Humboldt ( $\mathrm{SCH}$ ) que se desplaza entre la costa y los $80^{\circ} \mathrm{W}$ aproximadamente, y está compuesto por flujos y contraflujos paralelos a la costa. En la capa superficial de 0-25 m se distingue el Agua Subtropical con alta temperatura y salinidad. Bajo los $20 \mathrm{~m}$ se encuentra el Agua Subantártica, con bajos valores de temperatura, un mínimo de salinidad y altos valores de oxígeno disuelto, que alcanza cerca de los $100 \mathrm{~m}$ de profundidad. Entre los 100 y 400 m se encuentra el Agua Ecuatorial Subsuperficial, caracterizada por su baja temperatura, alta salinidad y un mínimo de oxígeno (Sievers \& Silva, 1982; Strub et al., 1998).

La mayor parte de los estudios sobre el zooplancton de la zona de Mejillones se han centrado en el componente quitinoso, copépodos y eufáusidos (Escribano \& McLaren, 1999; Escribano \& Hidalgo, 2000; Escribano et al., 2000, 2001), pero existe un escaso conocimiento sobre el componente gelatinoso (Pagès et al., 2001). La presente investigación tiene como objetivos determinar la composición específica de medusas, sifonóforos y ctenóforos, colectados en una transecta costa-océano ubicada frente a la bahía de Mejillones; determinar sus fluctuaciones temporales y su distribución vertical durante un ciclo estacional de pescas planctónicas; y determinar el efecto de las fluctuaciones ambientales sobre la distribución de estos organismos gelatinosos.

\section{MATERIALES Y MÉTODOS}

\section{Zona de estudio}

Durante los años 2000 y 2001 se efectuaron cuatro cruceros estacionales (octubre 2000, y enero, agosto y octubre 2001), frente a la bahía de Mejillones $\left(23^{\circ} \mathrm{S}\right)$. En cada crucero se efectuó una transecta de 12 millas de longitud, compuesta por tres estaciones oceanográficas ubicadas en la bahía (Estación 1), en el quiebre de la plataforma continental (Esta- 
ción 2) y en el talud (Estación 3) (Fig. 1). En cada estación se tomaron muestras estratificadas de zooplancton de día y de noche con redes WP-2 de $57 \mathrm{~cm}$ de diámetro de boca y $200 \mu \mathrm{m}$ de abertura de malla, provistas de flujómetros para la estimación del volumen de agua filtrado. Los estratos de pesca fueron de $0-25,25-50$, 50-100 y desde 100 hasta el fondo, con un máximo de $180 \mathrm{~m}$ de profundidad. Además, se realizó un estrato muy somero de espesor variable de acuerdo a la época del año, localizado entre la superficie del mar y la termoclina.

Las muestras se preservaron en agua de mar con formalina al $5 \%$, neutralizada con tetraborato de sodio. De cada muestra se identificaron y contaron todos los organismos gelatinosos. Dada la estructura polimórfica de los sifonóforos, en fisonéctidos se contó el número de nectóforos en la muestra y se dividió por el número de nectóforos atribuido a una especie y en calicóforos se contaron las fases poligástrica y eudoxia. En el análisis de la distribución estacional y vertical se consideraron las especies dominantes (> $5 \%$ de la abundancia de cada taxon), en a lo menos dos de los cuatro cruceros realizados. Para la abundancia estacional se consideraron los valores integrados en la columna de agua y para la distribución vertical la cantidad de individuos por estrato, expresada en ambos casos como individuos por $100 \mathrm{~m}^{-3}$.

En cada crucero se obtuvieron perfiles verticales de temperatura, salinidad y oxígeno disuelto con un CTD SeaBird SBE-19 desde un máximo de $180 \mathrm{~m}$ a superficie, o desde cerca del fondo en las estaciones más someras. Además, en cada estación se determinó la profundidad de la capa de mínimo de oxígeno (CMO), definida por la isolínea de $1 \mathrm{ml} \mathrm{O} \cdot \mathrm{L}^{-1}$. También, en cada período de muestreo se
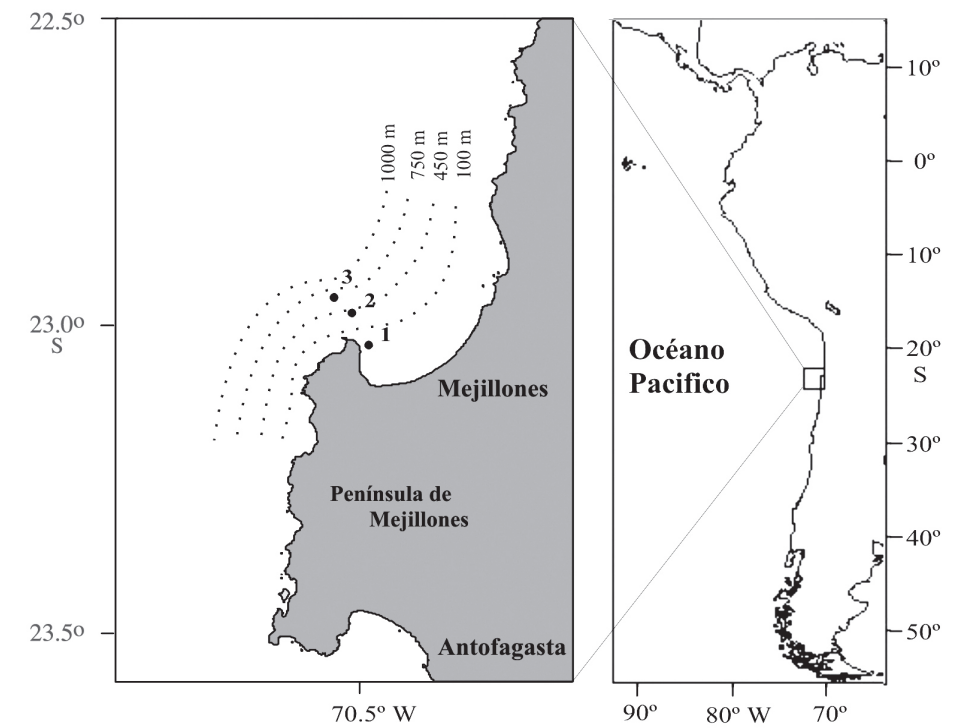

Figura 1. Ubicación de las estaciones oceanográficas frente a la bahía de Mejillones.

Figure 1. Location of oceanographic sampling stations off Mejillones bay. obtuvieron imágenes satelitales de temperatura superficial del mar (TSM), que cubrieron desde los 22,5 a $24^{\circ} \mathrm{S}$, tomadas por el satélite NOAA-9 y procesadas en la Escuela de Ciencias del Mar de la Pontificia Universidad Católica de Valparaíso.

Para analizar las relaciones existentes entre las especies dominantes y las variables ambientales, se utilizó la correlación de Pearson. Para disminuir la varianza, se aplicó la transformación $\ln (\mathrm{x}+1)$ a la abundancia relativa de cada especie. Para las variables ambientales se utilizó el promedio ponderado por estrato de profundidad, mediante la siguiente expresión:

$$
X_{i j}=\frac{\sum_{k=1} z_{j k} * C_{i j k}}{\sum z_{j k}}
$$

donde:

$\mathrm{X}_{\mathrm{ij}}=$ promedio del i-ésimo parámetro de la j-ésima estación.

$\mathrm{Z}_{\mathrm{ij}}=\mathrm{k}$-ésima profundidad de la $\mathrm{j}$ ésima estación.

$\mathrm{C}_{\mathrm{ijk}}=$ valor del i-ésimo parámetro en la j-ésima estación a la késima profundidad.

\section{RESULTADOS}

\section{Condiciones oceanográficas}

Mediante las imágenes satelitales de TSM obtenidas en los cuatro períodos de estudio se determinó un ciclo anual de temperatura superficial, con valores mínimos en octubre 2000 $\left(12-16^{\circ} \mathrm{C}\right) \mathrm{y}$ agosto $2001\left(13-16^{\circ} \mathrm{C}\right)$, y máximos en febrero $\left(18-23^{\circ} \mathrm{C}\right) \mathrm{y}$ octubre de 2001 (16-18 $\left.{ }^{\circ} \mathrm{C}\right)$. Los mínimos superficiales registrados en octubre de 2000 y agosto de 2001 se debieron a eventos de surgencia causados por vientos del S y SW, caracterizados por la presencia de aguas de baja temperatura en la franja cos- 
tera $\left(12-13^{\circ} \mathrm{C}\right)$ y aguas más cálidas en la zona oceánica. Durante estos eventos, al interior de las bahías de Mejillones y Antofagasta, se observaron aguas de mayor temperatura $\left(>14^{\circ} \mathrm{C}\right)$ correspondientes a la denominada sombra de surgencia, constituida por aguas más cálidas $\left(>2^{\circ} \mathrm{C}\right)$ que el resto del área afectada por la surgencia (Fig. 2).

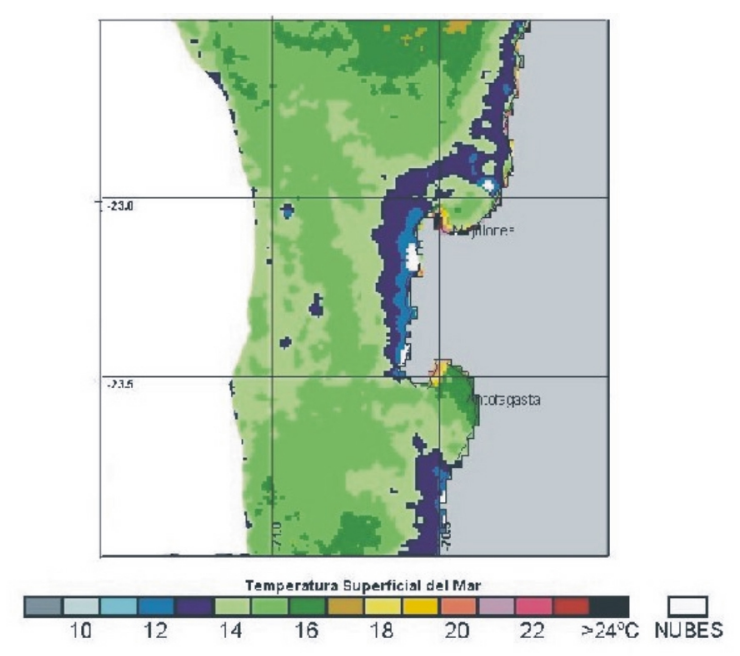

17 octubre 2000



5 agosto 2001
Las secciones verticales de los parámetros hidrográficos mostraron en octubre de 2000 un sistema de dos capas. Una capa superficial de 0-30 m con temperaturas mayores de $13^{\circ} \mathrm{C}$ y una termoclina bien desarrollada; salinidades bajas, particularmente en la estación oceánica $(<34,6$ psu), y altos valores de oxígeno disuelto en superficie $\left(>5 \mathrm{ml} \cdot \mathrm{L}^{-1}\right)$, que disminuyeron bruscamente a menos de $3 \mathrm{ml} \cdot \mathrm{L}^{-1}$



7 febrero 2001



19 octubre 2001

Figura 2. Imágenes satelitales de temperatura superficial del mar (TSM) frente a la península de Mejillones en cada período de muestreo.

Figure 2. Satellite images of sea surface temperature (SST) off Mejillones peninsula for each sampling period. 
bajo los $25 \mathrm{~m}$ de profundidad. La capa subsuperficial de 25-150 m fue más homogénea y se caracterizó por una disminución gradual de la temperatura, de $13^{\circ}$ a $12^{\circ} \mathrm{C}$; mayores valores de salinidad (> 34,8 psu) y bajos valores de oxígeno disuelto, inferiores a $0,5 \mathrm{ml} \cdot \mathrm{L}^{-1}$ bajo los $50 \mathrm{~m}$ de profundidad (Fig. 3a).

Esta estructura de dos capas se mantuvo en el resto de los períodos de muestreo, aunque con variaciones en el espesor de la capa superficial y en los valores de los parámetros abióticos. En febrero de 2001, las temperaturas fueron más altas y alcanzaron los $18^{\circ} \mathrm{C}$ en superficie, la termoclina fue más superficial y la temperatura más homogénea bajo los $50 \mathrm{~m}$. La salinidad superficial fue muy baja, con valores inferiores a 34,4 psu en los primeros $50 \mathrm{~m}$ y luego se incrementó en profundidad, alcanzando los 34,8 psu. La capa superficial fue bien oxigenada, alcanzando un máximo de $6 \mathrm{ml} \cdot \mathrm{L}^{-1}$ en los primeros $5 \mathrm{~m}$ en la estación 1, por debajo de los $60 \mathrm{~m}$ se registraron valores mínimos $\left(<1 \mathrm{ml} \cdot \mathrm{L}^{-1}\right)$ (Fig. 3b).

En agosto, se registraron las temperaturas más bajas del año, con valores inferiores a $15^{\circ} \mathrm{C}$ en superficie; la termoclina estaba poco desarrollada y era muy superficial $(5-15 \mathrm{~m})$ y la temperatura fue muy uniforme bajo los $15 \mathrm{~m}$. La salinidad presentó bajos valores superficiales y por debajo de los $25 \mathrm{~m}$ mostró una estructura muy homogénea, con valores de 34,9-35,0 psu. La concentración de oxígeno disuelto fue baja en la capa superficial en comparación con los demás períodos del año, con valores de $3 \mathrm{ml} \cdot \mathrm{L}^{-1}$ a $10 \mathrm{~m}$ de profundidad y valores mínimos por debajo de los $30 \mathrm{~m}$ en toda la transecta (Fig. 4a).

En octubre de 2001 las temperaturas fueron más altas que en octubre de 2000, registrándose un incremento en la profundidad de la termoclina desde la bahía hacia el océano. En superficie la temperatura alcanzó hasta los $18^{\circ} \mathrm{C}$ y fue más homogénea en profundidad, a partir de los $60 \mathrm{~m}$. La salinidad fue bastante homogénea en toda la columna de agua, con valores que fluctuaron entre 34,7 y 34, 9 psu, a excepción de un núcleo de menor salinidad registrado a los 25-30 m en la estación 3. La concentración de oxígeno disuelto presentó altos valores superficiales $\left(>5 \mathrm{ml} \cdot \mathrm{L}^{-1}\right)$, observándose un rápido descenso a partir de los $20 \mathrm{~m}$ en la zona costera; y bajo los $50 \mathrm{~m}$ predominaron concentraciones inferiores a $1 \mathrm{ml} \cdot \mathrm{L}^{-1}$ (Fig. 4b).

En el área de estudio se determinó la presencia de tres masas de agua, Agua Subtropical (AST), Agua Subantártica (ASAA) y Agua Ecuatorial
Subsuperficial (AESS), con un predominio de esta última en la columna de agua durante los distintos períodos analizados. La capa superficial (0-25 m) se caracterizó por una mezcla entre estas tres masas de agua, siendo predominante el AST en los primeros $10 \mathrm{~m}$, a excepción de octubre de 2000, donde apareció como un núcleo cálido al interior de la bahía. Entre los 40 y 140 m de profundidad, la masa predominante fue el AESS (Fig. 5).

\section{Composición taxonómica}

Se determinó un total de 44 especies carnívoras gelatinosas distribuidas en 23 de sifonóforos, 17 de hidromedusas y 4 de ctenóforos, 8 de las cuales se registran por primera vez en aguas chilenas del $\mathrm{SCH}$ (Tabla 1). Las especies más abundantes fueron los sifonóforos Muggiaea atlantica y Sphaeronectes gracilis, las hidromedusas Aglaura hemistoma, Cunina peregrina, Liriope tetraphylla, Obelia spp. y Solmundella bitentaculata, y los ctenóforos Pleurobrachia bachei y Thalassocalyce inconstans.

\section{Abundancia estacional}

La distribución temporal del macroplancton gelatinoso mostró variaciones estacionales estadísticamente significativas $(\mathrm{p}<0,05)$, con máximos de abundancia en primavera-verano y mínimos en el invierno. Este patrón de distribución temporal fue seguido por la mayoría de las especies dominantes (Tabla 1).

Los sifonóforos Muggiaea atlantica y Sphaeronectes gracilis, se colectaron en grandes cantidades en todos los períodos del año, incluso en invierno (Fig. 6). Los máximos se registraron en primavera de 2001 y fueron de casi 13.000 y 3.000 ind. $100 \mathrm{~m}^{3}$, para $M$. atlantica y $S$. gracilis respectivamente (Tabla 1). En octubre de 2000, también fueron abundantes las colonias larvales y adultas de Agalma elegans y los nectóforos de Abylopsis tetragona, que en conjunto constituyeron el 17,1\% de los sifonóforos. En verano, se registró una alta concentración de Bassia bassensis (28,4\% de los sifonóforos), Sphaeronectes irregularis $(13,5 \%)$ y $S$. gracilis que duplicó su abundancia. En invierno se determinó un fuerte descenso en el número de especies y en la abundancia relativa. A excepción de $M$. atlantica y S. gracilis que constituyeron el $92 \%$ de los sifonóforos totales, la mayoría de las especies restantes estuvo ausente. En primavera de 2001 la densidad de individuos fue tres veces superior a la primavera de 2000, siendo las dos especies domi- 

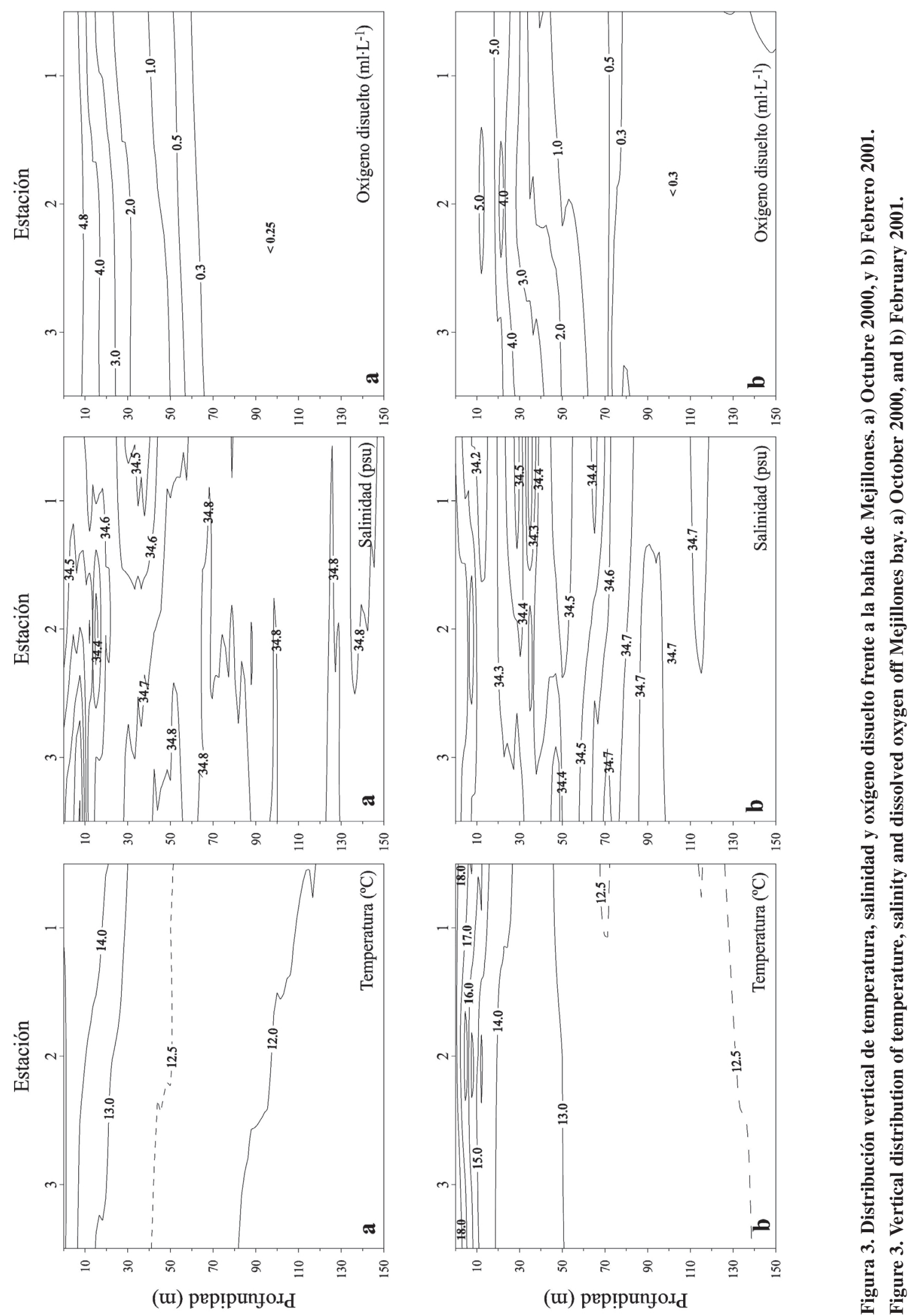

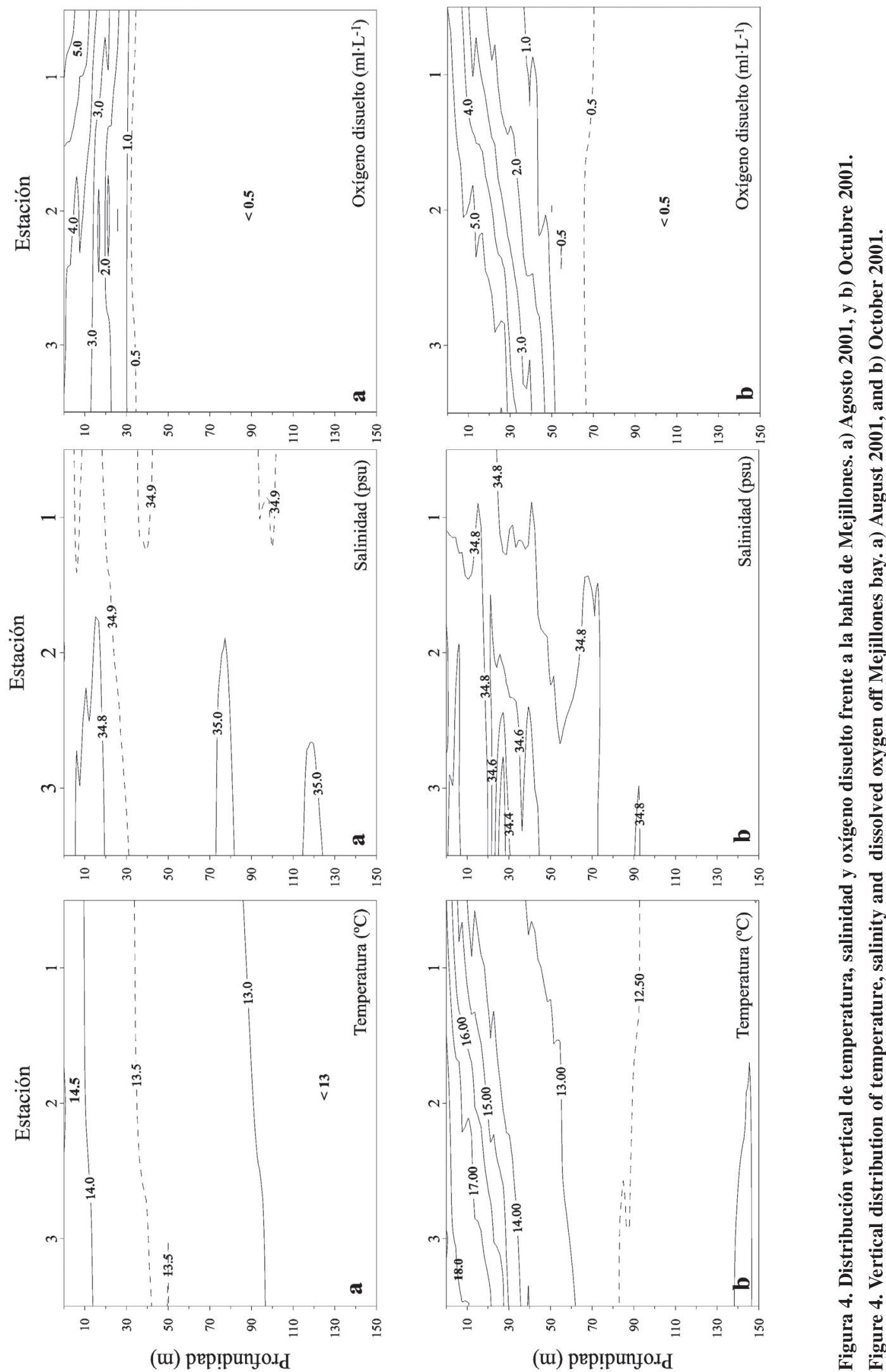

(w) peptpunjox

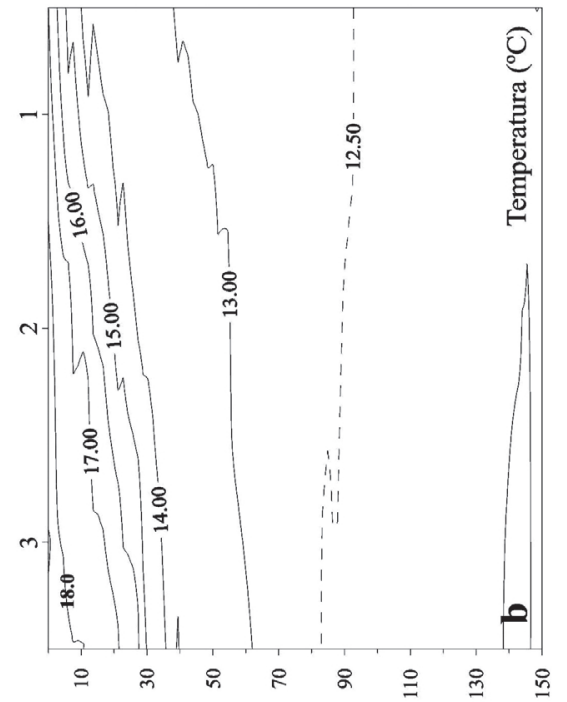

(u) peptpunyor 
Tabla 1. Densidad relativa (ind. $\cdot 100 \mathrm{~m}^{-3}$ ) de las especies identificadas frente a la bahía de Mejillones (octubre 2000-octubre 2001). El asterisco indica el primer registro para aguas del Sistema de la Corriente de Humboldt. DS: Desviación Estándar para 24 muestras por crucero. (FP): Fase Poligástrica, (FE): Fase Eudoxia.

Table 1. Relative density (ind. $\cdot 100 \mathrm{~m}^{-3}$ ) of the identified species off Mejillones bay (October 2000-October 2001). Asterisk indicate the first record for the Humboldt Current System. DS: Standard Deviation for 24 samples by cruise. (FP): Polygastric Phase, (FE): Eudoxid Phase.

\begin{tabular}{|c|c|c|c|c|c|c|c|c|c|c|c|c|c|c|c|c|}
\hline \multirow{2}{*}{$\begin{array}{l}\text { Crucero } \\
\text { Hydromedusae }\end{array}$} & \multicolumn{4}{|c|}{ Octubre 2000} & \multicolumn{4}{|c|}{ Febrero 2001} & \multicolumn{4}{|c|}{ Agosto 2001} & \multicolumn{4}{|c|}{ Octubre 2001} \\
\hline & Día & DS & Noche & DS & Día & DS & Noche & DS & Día & DS I & Noche & DS & Día & DS & Noche & e DS \\
\hline Aglaura hemistoma & 643 & 55 & 923 & 71 & 257 & 28 & 1795 & 157 & 0 & 0 & 11 & 2 & 4596 & 981 & 5993 & 1087 \\
\hline Obelia spp. & 615 & 84 & 101 & 13 & 3849 & 651 & 2623 & 393 & 2576 & 431 & 327 & 33 & 19 & 11 & 1426 & 823 \\
\hline Solmundella bitentaculata & 1532 & 154 & 423 & 40 & 149 & 21 & 756 & 103 & 67 & 10 & 20 & 3 & 3266 & 443 & 3837 & 803 \\
\hline Cunina peregrina & 597 & 65 & 815 & 86 & 425 & 62 & 1584 & 130 & 59 & 9 & 14 & 4 & 72 & 16 & 243 & 79 \\
\hline Liriope tetraphylla & 355 & 49 & 250 & 41 & 338 & 37 & 568 & 48 & 246 & 21 & 277 & 45 & 187 & 30 & 318 & 66 \\
\hline Rhopalonema velatum & 223 & 26 & 285 & 43 & 187 & 22 & 445 & 29 & 93 & 16 & 0 & 0 & 378 & 71 & 481 & 126 \\
\hline Clytia spp. & 641 & 92 & 199 & 43 & 21 & 6 & 125 & 16 & 27 & 5 & 7 & 2 & 131 & 70 & 8 & 5 \\
\hline Amphogona apicata $*$ & 62 & 9 & 45 & 7 & 32 & 8 & 37 & 5 & 14 & 3 & 3 & 1 & 124 & 55 & 62 & 21 \\
\hline Sarsia eximia & 5 & 1 & 0 & 0 & 68 & 20 & 78 & 15 & 26 & 5 & 0 & 0 & 6 & 3 & 58 & 33 \\
\hline Phialella quadrata & 85 & 16 & 0 & 0 & 34 & 10 & 0 & 0 & 0 & 0 & 0 & 0 & 19 & 11 & 24 & 14 \\
\hline Ectopleura dumortieri & 45 & 7 & 0 & 0 & 0 & 0 & 17 & 5 & 8 & 2 & 0 & 0 & 7 & 4 & 8 & 5 \\
\hline Sarsia coccometra * & 12 & 2 & 11 & 3 & 0 & 0 & 19 & 5 & 0 & 0 & 0 & 0 & 0 & 0 & 0 & 0 \\
\hline Euphysa aurata & 13 & 3 & 0 & 0 & 0 & 0 & 0 & 0 & 8 & 2 & 8 & 2 & 7 & 4 & 0 & 0 \\
\hline Proboscidactyla stellata $*$ & 0 & 0 & 0 & 0 & 0 & 0 & 8 & 2 & 0 & 0 & 0 & 0 & 6 & 3 & 0 & 0 \\
\hline Halitrephes maasi & 0 & 0 & 12 & 3 & 0 & 0 & 0 & 0 & 0 & 0 & 0 & 0 & 0 & 0 & 0 & 0 \\
\hline Leuckartiara zacae & 0 & 0 & 0 & 0 & 0 & 0 & 0 & 0 & 8 & 2 & 0 & 0 & 0 & 0 & 0 & 0 \\
\hline $\begin{array}{l}\text { Sminthea eurygaster } \\
\text { Siphonophora }\end{array}$ & 0 & 0 & 0 & 0 & 0 & 0 & 0 & 0 & 0 & 0 & 0 & 0 & 5 & 3 & 0 & 0 \\
\hline Abylopsis tetragona $(F P)$ & 362 & 39 & 12 & 3 & 23 & 3 & 53 & 7 & 0 & 0 & 0 & 0 & 49 & 8 & 72 & 35 \\
\hline Abylopsis tetragona (FE) & 185 & 28 & 86 & 9 & 171 & 17 & 301 & 34 & 0 & 0 & 0 & 0 & 180 & 50 & 238 & 37 \\
\hline Agalma elegans (colonia adulta) & 549 & 15 & 0 & 0 & 0 & 0 & 19 & 5 & 0 & 0 & 0 & 0 & 99 & 32 & 261 & 82 \\
\hline Agalma elegans (colonia larval) & 337 & 70 & 97 & 17 & 110 & 15 & 193 & 14 & 0 & 0 & 30 & 6 & 51 & 29 & 40 & 17 \\
\hline Agalma elegans $(F P)$ & 922 & 154 & 376 & 61 & 0 & 0 & 0 & 0 & 0 & 0 & 3 & 1 & 516 & 157 & 1017 & 423 \\
\hline Amphicaryon acaule (FP) & 12 & 2 & 12 & 3 & 8 & 2 & 78 & 14 & 0 & 0 & 0 & 0 & 54 & 12 & 47 & 15 \\
\hline Amphicaryon acaule (FE) & 0 & 0 & 37 & 10 & 0 & 0 & 9 & 2 & 0 & 0 & 0 & 0 & 41 & 11 & 2 & 1 \\
\hline Amphycarion ernesti (FP) & 0 & 0 & 0 & 0 & 7 & 2 & 0 & 0 & 0 & 0 & 0 & 0 & 3 & 2 & 0 & 0 \\
\hline Bassia bassensis $(F P)$ & 207 & 43 & 0 & 0 & 1520 & 193 & 934 & 141 & 43 & 12 & 14 & 4 & 143 & 62 & 53 & 25 \\
\hline Bassia bassensis (FE) & 237 & 56 & 25 & 7 & 2324 & 261 & 2375 & 270 & 69 & 15 & 19 & 4 & 253 & 117 & 180 & 13 \\
\hline Diphyes bojani (FE) & 0 & 0 & 0 & 0 & 33 & 5 & 11 & 3 & 0 & 0 & 0 & 0 & 0 & 0 & 0 & 0 \\
\hline Enneagonum hyalinum (FP) & 0 & 0 & 0 & 0 & 40 & 5 & 64 & 7 & 0 & 0 & 0 & 0 & 6 & 3 & 0 & 0 \\
\hline Enneagonum hyalinum (FE) & 8 & 2 & 0 & 0 & 26 & 3 & 32 & 5 & 0 & 0 & 0 & 0 & 12 & 7 & 7 & 3 \\
\hline Eudoxoides spiralis $(F P)$ & 61 & 14 & 11 & 3 & 2 & 0 & 6 & 1 & 0 & 0 & 0 & 0 & 29 & 9 & 7 & 4 \\
\hline Eudoxoides spiralis (FE) & 0 & 0 & 0 & 0 & 8 & 2 & 0 & 0 & 0 & 0 & 0 & 0 & 17 & 10 & 16 & 5 \\
\hline Fase eudoxia sp. & 0 & 0 & 0 & 0 & 0 & 0 & 0 & 0 & 0 & 0 & 0 & 0 & 12 & 7 & 0 & 0 \\
\hline Fase poligástrica $\mathrm{sp}$. & 0 & 0 & 0 & 0 & 0 & 0 & 0 & 0 & 0 & 0 & 0 & 0 & 3 & 2 & 0 & 0 \\
\hline Lensia ajax $(F P)$ & 0 & 0 & 0 & 0 & 13 & 3 & 6 & 2 & 0 & 0 & 0 & 0 & 1 & 1 & 0 & 0 \\
\hline Lensia ajax $(F E)$ & 0 & 0 & 0 & 0 & 21 & 6 & 2 & 1 & 0 & 0 & 0 & 0 & 0 & 0 & 0 & 0 \\
\hline Lensia campanella $(F P)$ & 0 & 0 & 0 & 0 & 0 & 0 & 11 & 3 & 0 & 0 & 0 & 0 & 0 & 0 & 0 & 0 \\
\hline Lensia challengeri (FP) & 54 & 9 & 11 & 3 & 58 & 6 & 89 & 10 & 65 & 18 & 3 & 1 & 167 & 44 & 148 & 34 \\
\hline Lensia challengeri (FE) & 75 & 11 & 22 & 6 & 116 & 14 & 340 & 42 & 0 & 0 & 0 & 0 & 544 & 47 & 355 & 104 \\
\hline Lensia cossack $(F P)$ & 0 & 0 & 0 & 0 & 0 & 0 & 0 & 0 & 0 & 0 & 0 & 0 & 0 & 0 & 4 & 3 \\
\hline Lensia fowleri $(F P)$ & 0 & 0 & 12 & 3 & 0 & 0 & 0 & 0 & 0 & 0 & 0 & 0 & 0 & 0 & 0 & 0 \\
\hline Lensia fowleri $(F E)$ & 0 & 0 & 25 & 7 & 0 & 0 & 2 & 1 & 0 & 0 & 0 & 0 & 0 & 0 & 0 & 0 \\
\hline Lensia hotspur $(F P)$ & 0 & 0 & 16 & 3 & 0 & 0 & 0 & 0 & 0 & 0 & 0 & 0 & 0 & 0 & 19 & 11 \\
\hline Lensia hotspur (FE) & 0 & 0 & 5 & 1 & 0 & 0 & 0 & 0 & 0 & 0 & 0 & 0 & 0 & 0 & 0 & 0 \\
\hline Lensia subtilis (FP) & 0 & 0 & 0 & 0 & 10 & 3 & 2 & 1 & 0 & 0 & 0 & 0 & 0 & 0 & 2 & 1 \\
\hline Muggiaea atlantica $(F P)$ & 2414 & 279 & 953 & 103 & 1635 & 126 & 1935 & 183 & 1413 & 155 & 329 & 33 & 7106 & 1737 & 75649 & 1331 \\
\hline Muggiaea atlantica (FE) & 5789 & 639 & 1459 & 162 & 1024 & 95 & 1163 & 104 & 1021 & 108 & 502 & 61 & 7099 & 1607 & 5978 & 1176 \\
\hline Praya dubia $(F P)$ & 0 & 0 & 0 & 0 & 2 & 0 & 0 & 0 & 0 & 0 & 0 & 0 & 0 & 0 & 0 & 0 \\
\hline Rhizophysa eysenhardti $(C) *$ & 8 & 2 & 0 & 0 & 0 & 0 & 0 & 0 & 0 & 0 & 0 & 0 & 0 & 0 & 38 & 22 \\
\hline Rosacea plicata $(F P)$ & 0 & 0 & 0 & 0 & 0 & 0 & 9 & 3 & 0 & 0 & 0 & 0 & 53 & 21 & 55 & 18 \\
\hline Rosacea plicata $(F E)$ & 57 & 8 & 122 & 30 & 143 & 15 & 79 & 8 & 11 & 3 & 0 & 0 & 14 & 8 & 21 & 7 \\
\hline Sphaeronectes fragilis $(F P)$ & 0 & 0 & 0 & 0 & 5 & 2 & 0 & 0 & 0 & 0 & 0 & 0 & 2 & 1 & 0 & 0 \\
\hline Sphaeronectes gracilis (FP) & 349 & 62 & 217 & 43 & 261 & 16 & 888 & 58 & 85 & 14 & 151 & 20 & 339 & 42 & 661 & 196 \\
\hline Sphaeronectes gracilis (FE) & 203 & 30 & 73 & 17 & 198 & 36 & 603 & 75 & 0 & 0 & 0 & 0 & 558 & 91 & 2417 & 648 \\
\hline Sphaeronectes irregularis $(F P) *$ & 19 & 5 & 36 & 8 & 449 & 60 & 523 & 56 & 45 & 7 & 7 & 2 & 77 & 40 & 68 & 5 \\
\hline Sphaeronectes irregularis (FE) & 0 & 0 & 5 & 1 & 24 & 6 & 17 & 5 & 0 & 0 & 0 & 0 & 62 & 25 & 40 & 23 \\
\hline $\begin{array}{l}\text { Vogtia pentacantha }(F P) \\
\text { Ctenophora }\end{array}$ & 0 & 0 & 0 & 0 & 0 & 0 & 0 & 0 & 0 & 0 & 0 & 0 & 0 & 0 & 3 & 2 \\
\hline Pleurobrachia bachei $*$ & 15544 & 2712 & 21206 & 149 & 65 & 10 & 280 & 48 & 0 & 0 & 14 & 4 & 1018 & 336 & 1133 & 459 \\
\hline Thalassocalyce inconstans * & 422 & 64 & 12 & 3 & 345 & 28 & 1450 & 168 & 7 & 2 & 0 & 0 & 682 & 44 & 429 & 64 \\
\hline Beröe cucumis & 56 & 8 & 56 & 12 & 37 & 7 & 503 & 43 & 15 & 3 & 81 & 15 & 164 & 27 & 482 & 194 \\
\hline Velamen parallelum* & 0 & 0 & 0 & 0 & 0 & 0 & 0 & 0 & 0 & 0 & 0 & 0 & 0 & 0 & 8 & 5 \\
\hline
\end{tabular}


nantes las responsables de este fuerte incremento.

Durante el período de estudio se registró la presencia constante de eudoxias de Muggiaea atlantica y Bassia bassensis, con máximos de $M$. atlantica en primavera y $B$. bassensis en verano. Además, en primavera y verano se determinaron altas densidades de eudoxias pertenecientes a Abylopsis tetragona, Lensia challengeri, Sphaeronectes gracilis y Rosacea plicata (Tabla 1).

En los distintos periodos estacionales, especialmente en ambas primaveras, los máximos de Muggiaea atlantica ocurrieron en las estaciones más costeras, registrándose una notoria disminución en la estación oceánica. En forma similar, la abundancia de Sphaeronectes gracilis también disminuyó de costa a océano, a excepción del verano en que se detectó un aumento hacia el océano (Fig. 6).

Las mayores abundancias de medusas se registraron en primavera y verano de 2001, caracterizadas por altas agregaciones de Aglaura hemistoma, Cunina peregrina, Obelia spp. y Solmundella bitentaculata, coincidentes con los incrementos de temperaturas en esos periodos. Las mayores concentraciones de A. hemistoma y S. bitentaculata ocurrieron en primavera (10.588 y 7.103 ind $\cdot 100 \mathrm{~m}$ ${ }^{3}$ respectivamente), mientras que las de Obelia spp. y $C$. peregrina ocurrieron en verano (6.472 y 2.052 ind $100 \mathrm{~m}^{-3}$, respectivamente). En invierno, a excepción de Obelia spp. y Liriope tetraphylla, las medusas fueron muy escasas y varias especies colectadas en primavera y verano, estuvieron ausentes en este período (Tabla 1). En primavera de 2001, la densi-


Figura 5. Distribución porcentual de las masas de agua frente a la bahía de Mejillones. AST: Agua Subtropical, ASSA: Agua Subantártica y AESS: Agua Ecuatorial Subsuperficial.

Figure 5. Percentage distribution of water masses off Mejillones bay. AST: Subtropical Water, ASSA: Subantarctic Water and AESS: Equatorial Subsurface Water. 

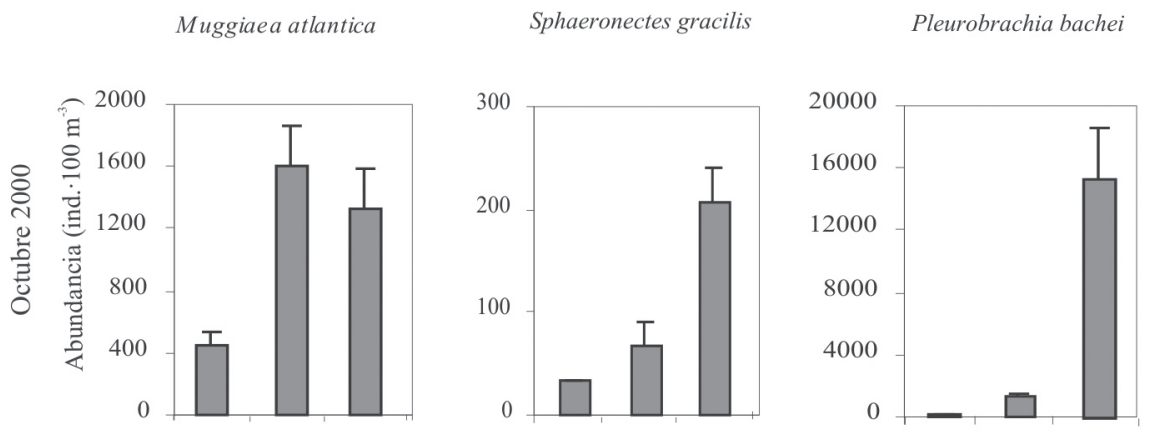

Thalassocalyce inconstans


\section{Estación}

Figura 6. Distribución espacio-temporal de las especies dominantes de sifonóforos y ctenóforos en las estaciones situadas frente a la bahía de Mejillones.

Figure 6. Seasonal and spatial distribution of dominant species of siphonophores and ctenophores in the stations off Mejillones bay. 
dad de individuos fue cuatro veces superior a la de primavera de 2000, debido a fuertes incrementos poblacionales de $S$. bitentaculata y A. hemistoma.

En primavera de 2000 la mayoría de las especies dominantes presentó un descenso de la abundancia en el gradiente costa-océano, a excepción de Cunina peregrina y Liriope tetraphylla que mostraron un comportamiento inverso. En verano, $C$. peregrina, L. tetraphylla, Obelia spp. y Solmundella bitentaculata presentaron una disminución en el gradiente costa-océano y solamente Aglaura hemistoma fue más abundante en la estación oceánica. En invierno, ocurrió un predominio de Obelia spp. (76\% de las medusas), con máximos en la estación oceánica. En octubre de 2001, se mantuvo el patrón de disminución costa-océano en las especies dominantes (Fig. 7).

Con respecto a los ctenóforos, las mayores concentraciones se registraron en primavera de 2000 , debidas a Pleurobrachia bachei que alcanzó un máximo de 16.750 ind. $100 \mathrm{~m}^{-3}$. En verano disminuyó drásticamente la abundancia de $P$. bachei, siendo reemplazada por Beroe cucumis y Thalassocalyce inconstans, alcanzando esta última su máximo estacional (1.795 ind. $100 \mathrm{~m}^{-3}$ ). En invierno, al igual que en sifonóforos y medusas, se observó una fuerte disminución de abundancia, con un total de 110 ind. $100 \mathrm{~m}^{-3}$. En primavera de 2001 , nuevamente se registraron altas densidades de ctenóforos, con dominancia de $P$. bachei sobre $T$. inconstans (Tabla 1).

En los diferentes períodos del año, Pleurobrachia bachei se distribuyó en el interior de la bahía, siendo muy escasa en las estaciones 2 y 3. En cambio, Thalassocalyce inconstans extendió su distribución hasta el quiebre de la plataforma (Est. 1 y 2) y su abundancia decreció hacia el área oceánica (Fig. 6).

\section{Distribución vertical}

Los sifonóforos se encontraron en los primeros 100 $\mathrm{m}$ de profundidad. Las especies dominantes, Muggiaea atlantica y Sphaeronectes gracilis presentaron una distribución vertical similar, pues generalmente se capturaron en los primeros $50 \mathrm{~m}$, por encima de la CMO. Las mayores abundancias se determinaron en el estrato superficial (0-25 m), particularmente sobre la termoclina, tanto de día como de noche, no observándose patrones de migración vertical. Solamente en la estación 2 ambas especies se colectaron por debajo de los $100 \mathrm{~m}$. Como las dos especies tuvieron un patrón similar de distribución batimétrica, solo se representó la distribución vertical de $M$. atlantica (Fig. 8). El análisis de correlación de Pearson, mostró que en general, $M$. atlantica y $S$. gracilis presentaron una relación directa significativa $(\mathrm{p}<0,05)$ con la temperatura y el contenido de oxígeno disuelto, e inversa con sigma t (Tabla 2).

Al igual que los sifonóforos, las medusas también se encontraron en los primeros $100 \mathrm{~m}$ de profundidad en los diferentes períodos del año, patrón de distribución vertical que se observó en todas las especies dominantes Aglaura hemistoma, Cunina peregrina, Liriope tetraphylla, Obelia spp. y Solmundella bitentaculata. Sin embargo, estas especies se distribuyeron en diferentes niveles de la columna de agua, así por ejemplo, Obelia spp. se colectó más superficialmente (0-25 m), por encima de la termoclina (Fig. 9). En cambio, A. hemistoma, C. peregrina, L. tetraphylla y S. bitentaculata se colectaron en un mayor rango de distribución batimétrica $(0-100 \mathrm{~m})$, concentrándose en los primeros $50 \mathrm{~m}$, sobre la CMO, aunque ocasionalmente alcanzaron hasta los $200 \mathrm{~m}$. Al igual que en sifonóforos, no se registraron variaciones en la distribución vertical día-noche que denotaran algún patrón migratorio. Debido a que estas especies tuvieron un patrón de distribución similar, solo se representó la distribución vertical de L. tetraphylla (Fig. 10). El análisis de Pearson mostró que Obelia spp. y L. tetraphylla presentaron una correlación positiva con la temperatura $(\mathrm{p}<0,05)$, mientras que en $S$. bitentaculata fue negativa solo en octubre de 2001. Además, Obelia spp., L. tetraphylla y $S$. bitentaculata presentaron una correlación significativa $(\mathrm{p}<0,05)$ con el contenido de oxígeno disuelto (Tabla 2).

Pleurobrachia bachei y Talassocalyce inconstans se colectaron en los primeros $100 \mathrm{~m}$ de profundidad, pero sus máximos se registraron en los primeros $50 \mathrm{~m}$, por encima de la CMO (Fig. 11). En octubre del 2000, cuando ocurrió el evento de surgencia, ambas especies se concentraron en los niveles más superficiales $(0-25 \mathrm{~m})$. A diferencia de lo observado en sifonóforos y medusas, no se detectaron correlaciones significativas entre la abundancia de estas especies y los parámetros oceanográficos, además al igual que en ambos grupos no se observaron desplazamientos verticales día-noche (Tabla 2). 

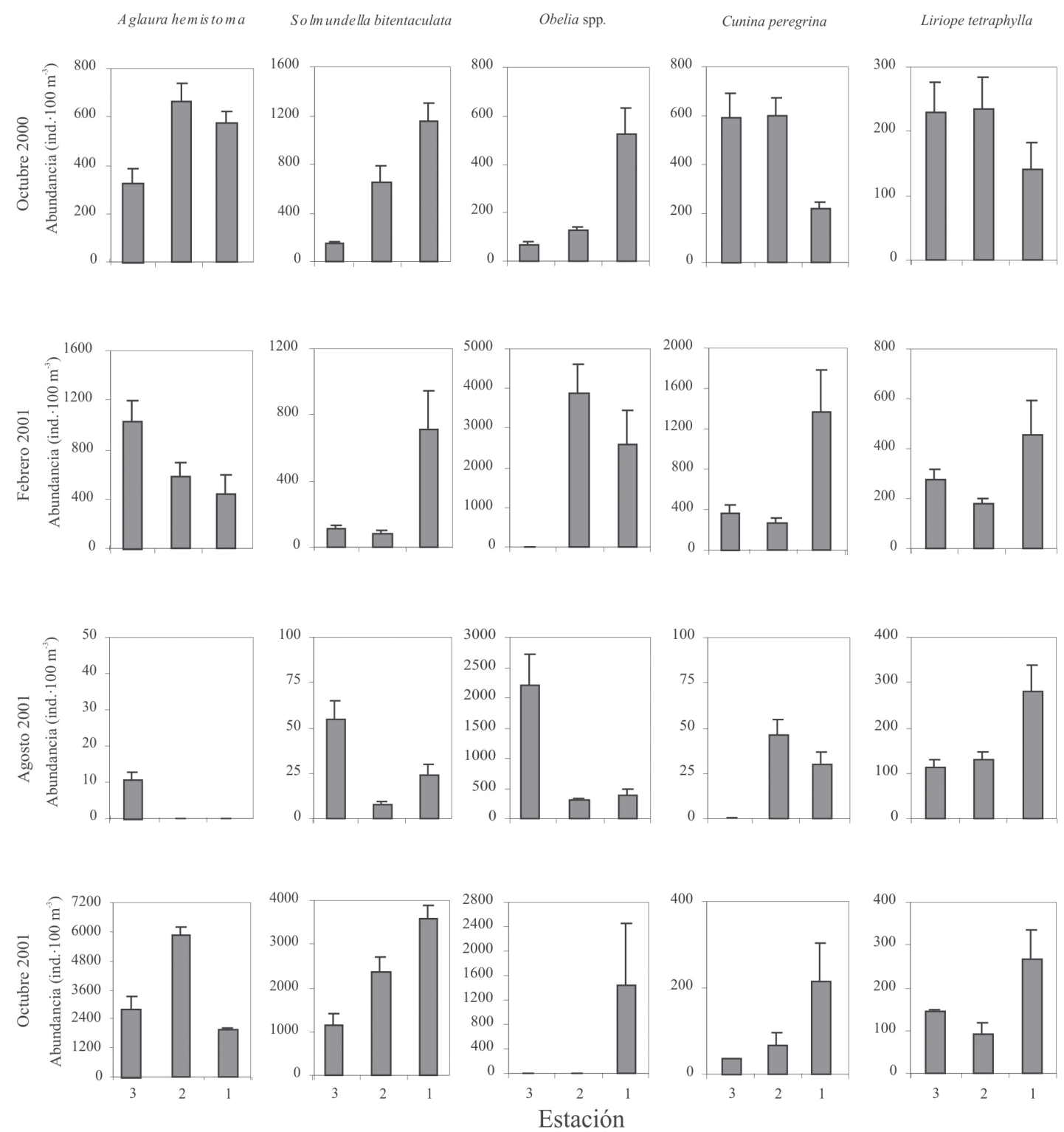

Figura 7. Distribución espacio-temporal de las especies dominantes de medusas en las estaciones situadas frente a la bahía de Mejillones.

Figure 7. Seasonal and spatial distribution of dominant species of medusae in the stations off Mejillones bay. 
Tabla 2. Valores de correlación entre las abundancias relativas y las variables ambientales para los distintos periodos de muestreo. En negrita se indican los valores significativos a un nivel $\mathbf{p}<\mathbf{0 , 0 5}$.

Table 2. Correlation values between the relative abundances and the environmental variables for the different periods of sampling. In bold the significative values are indicated at level $p<0.05$.

\begin{tabular}{|c|c|c|c|c|c|c|c|c|c|c|c|c|}
\hline Octubre 2000 & $\mathbf{T}$ & $\mathbf{S}$ & $\mathbf{O}$ & St & $\mathbf{A h}$ & Cp & Lt & Osp & Sb & Ma & $\mathrm{Sg}$ & $\mathbf{P b}$ \\
\hline $\mathrm{T}$ & 1 & & & & & & & & & & & \\
\hline $\mathrm{S}$ & $-0,79$ & 1,00 & & & & & & & & & & \\
\hline $\mathrm{O}$ & $\mathbf{0 , 8 5}$ & $-0,89$ & 1,00 & & & & & & & & & \\
\hline St & $-0,95$ & 0,92 & $-0,94$ & 1,00 & & & & & & & & \\
\hline $\mathrm{Ah}$ & 0,07 & 0,08 & 0,08 & 0,01 & 1,00 & & & & & & & \\
\hline $\mathrm{Cp}$ & 0,22 & $-0,01$ & 0,33 & $-0,20$ & 0,52 & 1,00 & & & & & & \\
\hline $\mathrm{Lt}$ & 0,37 & $-0,69$ & 0,71 & $-0,55$ & 0,28 & 0,26 & 1,00 & & & & & \\
\hline Osp & 0,72 & $-0,42$ & 0,61 & $-0,60$ & 0,43 & 0,35 & 0,11 & 1,00 & & & & \\
\hline $\mathrm{Sb}$ & 0,32 & $-0,10$ & $-0,09$ & $-0,19$ & 0,18 & 0,05 & $-0,39$ & 0,35 & 1,00 & & & \\
\hline Ma & 0,55 & $-0,29$ & 0,56 & $-0,46$ & 0,14 & 0,38 & 0,21 & $\mathbf{0 , 7 3}$ & $-0,01$ & 1,00 & & \\
\hline $\mathrm{Sg}$ & $\mathbf{0 , 8 0}$ & $-0,51$ & 0,67 & $-0,69$ & 0,30 & 0,46 & 0,33 & 0,72 & 0,36 & 0,70 & 1,00 & \\
\hline $\mathrm{Pb}$ & 0,56 & $-0,25$ & 0,31 & $-0,45$ & 0,50 & 0,51 & 0,21 & 0,31 & 0,53 & 0,09 & 0,66 & 1,00 \\
\hline Febrero 2001 & $\mathbf{T}$ & $\mathbf{S}$ & $\mathbf{O}$ & St & Ah & Cp & Lt & Osp & Sb & Ma & $\mathbf{S g}$ & \\
\hline \multicolumn{13}{|l|}{$\mathrm{Pb}$} \\
\hline $\mathrm{T}$ & 1,00 & & & & & & & & & & & \\
\hline $\mathrm{S}$ & $-0,82$ & 1,00 & & & & & & & & & & \\
\hline $\mathrm{O}$ & $\mathbf{0 , 8 9}$ & $-0,95$ & 1,00 & & & & & & & & & \\
\hline St & $-0,97$ & 0,92 & $-0,97$ & 1,00 & & & & & & & & \\
\hline $\mathrm{Ah}$ & $-0,54$ & 0,34 & $-0,38$ & 0,48 & 1,00 & & & & & & & \\
\hline $\mathrm{Cp}$ & 0,30 & $-0,06$ & 0,13 & $-0,21$ & 0,18 & 1,00 & & & & & & \\
\hline $\mathrm{Lt}$ & 0,47 & $-0,51$ & 0,53 & $-0,53$ & $-0,78$ & $-0,46$ & 1,00 & & & & & \\
\hline Osp & $\mathbf{0 , 7 3}$ & $-0,57$ & 0,70 & $-0,69$ & $-0,32$ & 0,51 & 0,22 & 1,00 & & & & \\
\hline $\mathrm{Sb}$ & $-0,03$ & 0,37 & $-0,28$ & 0,20 & $-0,56$ & $-0,09$ & 0,25 & 0,19 & 1,00 & & & \\
\hline Ma & 0,55 & $-0,75$ & 0,75 & $-0,68$ & $-0,09$ & 0,41 & 0,27 & 0,49 & $-0,53$ & 1,00 & & \\
\hline $\mathrm{Sg}$ & 0,63 & $-0,62$ & 0,71 & $-0,69$ & $-0,37$ & 0,30 & 0,29 & 0,50 & $-0,37$ & 0,73 & 1,00 & \\
\hline $\mathrm{Pb}$ & $-0,47$ & 0,49 & $-0,52$ & 0,47 & $-0,04$ & 0,07 & $-0,04$ & $-0,59$ & 0,01 & $-0,03$ & $-0,08$ & 1,00 \\
\hline Agosto 2001 & $\mathbf{T}$ & $\mathbf{S}$ & $\mathbf{O}$ & St & Lt & Osp & Sb & Ma & $\mathrm{Sg}$ & & & \\
\hline $\mathrm{T}$ & 1,00 & & & & & & & & & & & \\
\hline $\mathrm{S}$ & $-0,81$ & 1,00 & & & & & & & & & & \\
\hline $\mathrm{O}$ & $\mathbf{0 , 8 8}$ & $-0,57$ & 1,00 & & & & & & & & & \\
\hline St & $-0,98$ & $\mathbf{0 , 8 7}$ & $-0,83$ & 1,00 & & & & & & & & \\
\hline $\mathrm{Lt}$ & $\mathbf{0 , 8 0}$ & $-0,64$ & 0,62 & $-0,81$ & 1,00 & & & & & & & \\
\hline Osp & $\mathbf{0 , 8 8}$ & $-0,80$ & 0,86 & $-0,87$ & 0,47 & 1,00 & & & & & & \\
\hline $\mathrm{Sb}$ & 0,54 & $-0,26$ & 0,70 & $-0,52$ & 0,28 & 0,62 & 1,00 & & & & & \\
\hline Ma & 0,84 & $-0,60$ & 0,87 & $-0,84$ & 0,66 & $\mathbf{0 , 8 0}$ & 0,69 & 1,00 & & & & \\
\hline $\mathrm{Sg}$ & $\mathbf{0 , 8 4}$ & $-0,62$ & $\mathbf{0 , 8 3}$ & $-0,81$ & $\mathbf{0 , 8 7}$ & 0,64 & 0,48 & $\mathbf{0 , 7 5}$ & 1,00 & & & \\
\hline Octubre 2001 & $\mathbf{T}$ & $\mathbf{S}$ & $\mathbf{O}$ & St & Ah & Cp & Lt & Osp & Sb & Мa & $\mathbf{S g}$ & $\mathbf{P b}$ \\
\hline $\mathrm{T}$ & 1,00 & & & & & & & & & & & \\
\hline $\mathrm{S}$ & $-0,01$ & 1,00 & & & & & & & & & & \\
\hline $\mathrm{O}$ & $\mathbf{0 , 9 7}$ & $-0,21$ & 1,00 & & & & & & & & & \\
\hline St & $-0,96$ & 0,28 & $-0,99$ & 1,00 & & & & & & & & \\
\hline $\mathrm{Ah}$ & 0,12 & $-0,10$ & 0,12 & $-0,13$ & 1,00 & & & & & & & \\
\hline $\mathrm{Cp}$ & $-0,64$ & 0,11 & $-0,62$ & 0,63 & $-0,04$ & 1,00 & & & & & & \\
\hline $\mathrm{Lt}$ & 0,76 & $-0,07$ & $\mathbf{0 , 7 0}$ & $-\mathbf{0 , 7 3}$ & 0,22 & $-0,36$ & 1,00 & & & & & \\
\hline Osp & $-0,15$ & 0,07 & $-0,10$ & 0,07 & $-0,46$ & 0,04 & $-0,10$ & 1,00 & & & & \\
\hline $\mathrm{Sb}$ & $-0,60$ & 0,35 & $-0,65$ & 0,64 & $-0,08$ & 0,54 & $-0,40$ & 0,43 & 1,00 & & & \\
\hline $\mathrm{Ma}$ & 0,06 & 0,34 & 0,06 & $-0,06$ & 0,32 & 0,00 & 0,14 & 0,57 & 0,24 & 1,00 & & \\
\hline $\mathrm{Sg}$ & 0,59 & 0,06 & 0,57 & $-0,56$ & 0,43 & $-0,08$ & 0,68 & $-0,12$ & $-0,48$ & 0,37 & 1,00 & \\
\hline $\mathrm{Pb}$ & $-0,42$ & 0,27 & $-0,35$ & 0,38 & $-0,07$ & 0,37 & $-0,44$ & 0,67 & 0,52 & 0,74 & $-0,18$ & 1,00 \\
\hline
\end{tabular}





Figura 8. Distribución vertical diurna (blanco) y nocturna (negro) de Muggiaea atlantica en los diferentes períodos de muestreo. La barra gris corresponde a los organismos colectados sobre la termoclina. La línea segmentada indica la profundidad de la capa de mínimo oxígeno.

Figure 8. Day (white) and night (black) vertical distribution of Muggiaea atlantica in different sampling periods. The gray bar corresponds to the organisms collected above the thermocline. Segmented line indicates the depth of the minimum oxygen layer. 



Figura 9. Distribución vertical diurna (blanco) y nocturna (negro) de Obelia spp. en los diferentes períodos de muestreo. La barra gris corresponde a los organismos colectados sobre la termoclina. La línea segmentada indica la profundidad de la capa de mínimo oxígeno.

Figure 9. Day (white) and night (black) vertical distribution of Obelia spp. in different sampling periods. The gray bar corresponds to the organisms collected above the thermocline. Segmented line indicates the depth of the minimum oxygen layer. 

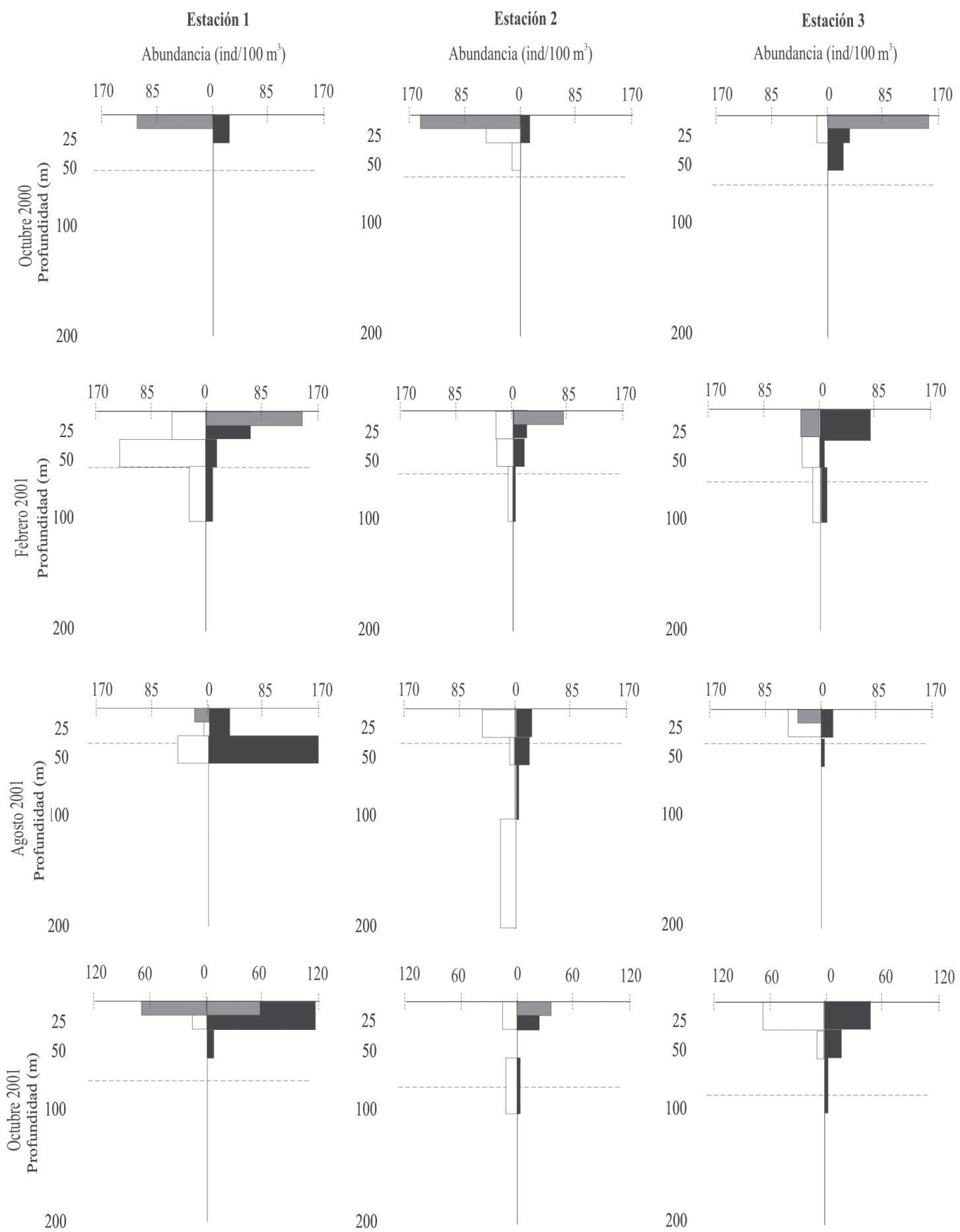

Figura 10. Distribución vertical diurna (blanco) y nocturna (negro) de Liriope tetraphylla en los diferentes períodos de muestreo. La barra gris corresponde a los organismos colectados sobre la termoclina. La línea segmentada indica la profundidad de la capa de mínimo oxígeno.

Figure 10. Day (white) and night (black) vertical distribution of Liriope tetraphylla in different sampling periods. The gray bar corresponds to the organisms collected above the thermocline. Segmented line indicates the depth of the minimum oxygen layer. 



Figura 11. Distribución vertical diurna (blanco) y nocturna (negro) de Pleurobrachia bachei en los diferentes períodos de muestreo. La barra gris corresponde a los organismos colectados sobre la termoclina. La línea segmentada indica la profundidad de la capa de mínimo oxígeno.

Figure 11. Day (white) and night (black) vertical distribution of Pleurobrachia bachei in different sampling periods. The gray bar corresponds to the organisms collected above the thermocline. Segmented line indicates the depth of the minimum oxygen layer. 


\section{DISCUSIÓN}

Los perfiles verticales de los parámetros oceanográficos obtenidos en la transecta costa-océano ubicada frente a la bahía de Mejillones, confirmaron la presencia de una estructura oceanográfica de dos capas, una superficial y otra profunda, la cual ha sido señalada anteriormente para esta zona (Rodríguez et al., 1991; Pagès et al., 2001). La fluctuación estacional de la capa superficial de 0-30 m, caracterizada por un calentamiento en verano y un enfriamiento en invierno, fue modificada en octubre de 2000 y agosto de 2001 por eventos de surgencia, que favorecieron el ascenso de aguas de menor temperatura $\left(12^{\circ} \mathrm{C}\right)$, generando un frente costero en el borde de la plataforma continental. En esta zona, los eventos de surgencia son comunes a lo largo del año y tienen mayor intensidad en primaveraverano (Rodríguez et al., 1991). La capa profunda (30-180 m), fue más homogénea, particularmente en invierno. En todos los períodos estacionales se registró la CMO bien desarrollada, centrada alrededor de 20-55 m, con valores mínimos de oxígeno (< $\left.1 \mathrm{ml} \cdot \mathrm{L}^{-1}\right)$, característicos de las aguas ecuatoriales subsuperficiales (Sievers \& Silva, 1982; Strub et al., 1998).

Los resultados obtenidos permiten incrementar el número de especies registradas en esta área, pues se identificaron 44 especies en comparación con las 32 registradas previamente frente a Antofagasta y Mejillones (Pagès et al., 2001). Esta diferencia fue más notoria en las medusas, donde el número de especies se incrementó de 7 a 17. Del total de especies determinadas, se agregaron 8 nuevos registros para aguas chilenas, correspondientes a 2 sifonóforos, 3 medusas y 3 ctenóforos (Tabla 1).

Además, se aclara la identificación de las especies del Phylum Ctenophora, grupo de compleja taxonomía y difícil preservación, que indica que la especie dominante en aguas chilenas citada como Pleurobrachia pileus por Palma (1994) y Palma \& Rosales (1995), corresponde a Pleurobrachia bachei, que a diferencia de $P$. pileus, tiene los bulbos tentaculares adosados a la faringe y al mismo nivel del esófago. Como así mismo, los especímenes identificados como Mnemiopsis sp. (Palma, 1994; Palma \& Rosales, 1995) y probablemente aquellos identificados como Ocyropsis sp. (Pagès et al., 2001), corresponden a Thalassocalyce inconstans. Esta especie se destruye fácilmente después de la captura y fue descrita hace pocos años sobre la base de 16 ejemplares obtenidos mediante buceo en el
Mar de los Sargazos (Madin \& Harbison, 1978). Se caracteriza por la presencia de ocho filas cortas de peines, de igual longitud, y por el curso sinuoso y largo de los canales paragástricos.

Los cambios estacionales del ambiente y la biología de las especies son los principales factores que afectan la dinámica poblacional y distribución estacional del macroplancton gelatinoso en aguas templadas (Gili et al., 1987; Palma \& Rosales, 1995). Además, se ha sugerido que las variaciones en la temperatura superficial del mar así como los eventos de surgencia, serían responsables de las máximas concentraciones de zooplancton en primavera y verano frente a la península de Mejillones (Escribano et al., 2000). Los máximos de abundancia registrados frente a la bahía de Mejillones, fueron causados por especies que pueden originar asexualmente decenas de medusas, como las de los géneros Clytia y Obelia; o los sifonóforos Abylopsis tetragona, Bassia bassensis y Muggiaea atlantica, cuyo número eudoxias fue muy elevado en primavera y verano (Tabla 1). Lo mismo ocurrió con el ctenóforo Pleurobrachia bachei que formó densas agregaciones (> $150 \mathrm{ind} \cdot \mathrm{m}^{-3}$ ) en el interior de la bahía, asociadas al evento de surgencia de octubre del 2000.

La cantidad de eudoxias de Muggiaea atlantica y Bassia bassensis colectadas en los diferentes períodos de muestreo, incluso en invierno, sugiere que en esta zona, estas especies tienen un período reproductivo más extenso que el resto de los sifonóforos (Tabla 1). Al respecto, estudios realizados por Escribano \& Hidalgo (2000), sugieren que las altas tasas de reproducción y crecimiento de los copépodos en la bahía de Mejillones, pueden ser utilizadas como una estrategia para mantener sus poblaciones en el interior de la bahía, dado que en períodos de surgencia activa, alrededor del 30\% de los ejemplares son advectados fuera de la bahía.

La abundancia de organismos gelatinosos registrada frente a la bahía de Mejillones, fue superior en un orden de magnitud a las observadas en la zona centro-sur por Palma (1994), Palma \& Rosales (1995) y Maturana (1999). Esta mayor abundancia se debería a los frecuentes eventos de surgencia que caracterizan la zona de Mejillones y que favorecen el desarrollo de altas tasas de producción primaria (Rodríguez et al., 1986; Marín et al., 1993; Santibáñez, 2003), esenciales para el mantenimiento de poblaciones de algunos herbívoros, como Calanus chilensis (Escribano \& McLaren, 1999).

En los diferentes períodos de muestreo, las ma- 
yores abundancias se localizaron en la bahía y en el quiebre de la plataforma (Figs. 6 y 7). Es probable que estos incrementos estén relacionados con la presencia de frentes térmicos en zonas de surgencia, que pueden generar áreas de retención de organismos planctónicos (Graham et al., 1992; Escribano et al., 2002; Marín et al., 2003), como también a la presencia de un giro ciclónico al interior de la bahía que puede aminorar su transporte mar afuera (Olivares, 2000; Santibáñez, 2003).

En la estación oceánica, se determinó en las distintas estaciones del año, un fuerte descenso en la abundancia de carnívoros gelatinosos, donde predominaron especies de características oceánicas, como Aglaura hemistoma, Liriope tetraphylla, Abylopsis tetragona y Bassia bassensis (Palma, 1977; Pagès et al., 2001). Es probable que este descenso esté asociado a una menor disponibilidad trófica, pues en esta estación también se registró una fuerte caída en la concentración de clorofila "a" y en la abundancia de copépodos (Santibáñez, 2003). La disminución gradual de abundancia de los cnidarios en el gradiente costa-océano es común en diferentes áreas marinas y parece relacionada también, con los procesos advectivos en la capa de Ekman, que transportan y dispersan los organismos fuera de la costa (Gili et al., 1988, 1991). Al respecto, Escribano et al., (2001) estimaron que el $22 \%$ de la biomasa de Calanus chilensis puede perderse fuera de la costa por transporte advectivo causado por la surgencia frente a Mejillones.

La distribución vertical del macroplancton gelatinoso, mostró que la mayoría de las especies colectadas corresponden a especies neríticas, comunes en los primeros $50 \mathrm{~m}$ de profundidad, como Muggiaea atlantica, Sphaeronectes gracilis, Obelia spp. y Pleurobrachia bachei. En cambio, especies como Aglaura hemistoma, Liriope tetraphylla, Solmundella bitentaculata, Abylopsis tetragona y Bassia bassensis de características más oceánicas, tuvieron una distribución batimétrica más amplia y se encontraron hasta los 150-180 m de profundidad.

Por otra parte, la CMO se detectó en aguas muy someras (20-50 m) en los distintos períodos de muestreo. Esta capa que generalmente ocurre por debajo de la termoclina, es característica frente a la península de Mejillones y puede penetrar hasta los 30-50 m cerca de la costa, limitando la distribución vertical de los organismos (Morales et al., 1996; Escribano et al., 2000, 2001). Los resultados obtenidos mostraron que la mayoría de las especies se localizaron en los primeros $50 \mathrm{~m}$ y estuvieron asociadas a aguas subtropicales y subantárticas, de mayor temperatura y mayor contenido de oxígeno disuelto, que las aguas ecuatoriales subsuperficiales que predominaron bajo los $50 \mathrm{~m}$ de profundidad (Fig. 5). Esto se reflejó en los resultados del análisis de Pearson, que indicaron que varias especies dominantes tuvieron una correlación positiva con la temperatura y el contenido de oxígeno disuelto, reduciendo su rango de distribución batimétrica a las capas bien oxigenadas (Figs. 8 a 11).

La mayor parte de los organismos gelatinosos colectados frente a Mejillones, son habitantes frecuentes en los primeros 100 m en aguas subantárticas del Sistema de la Corriente de Humboldt (Fagetti, 1973; Palma, 1994; Palma \& Rosales, 1995; Pagès et al., 2001). Sin embargo, la distribución vertical de los carnívoros gelatinosos frente a Mejillones estuvo limitada a los primeros 25-50 m debido a la presencia de la CMO. En este estrato, los organismos estuvieron asociados a aguas subantárticas y a su mezcla con aguas subtropicales, características de esta zona. La distribución vertical de varias de las especies determinadas frente a Mejillones es más amplia en aguas oceánicas de la costa central, ya que el límite superior de la CMO es más profundo y se encuentra alrededor de los $100 \mathrm{~m}$ de profundidad (Ulloa et al., 2000).

\section{CONCLUSIONES}

1. Los sifonóforos Sphaeronectes irregularis y Rhizophysa eysenhardti, las medusas Amphogona apicata, Proboscidactyla stellata y Sarsia coccometra, y los ctenóforos Pleurobrachia bachei, Thalassocalyce inconstans y Velamen parallelum se registran por primera vez para aguas costeras del Sistema de la Corriente de Humboldt.

2. Los carnívoros gelatinosos presentaron fuertes fluctuaciones estacionales de abundancia, con mínimos en invierno y máximos en primaveraverano, asociados a las variaciones de los parámetros oceanográficos y a eventos de surgencia costera.

3. La distribución vertical del macroplancton gelatinoso estuvo fuertemente influenciada por la profundidad de la termoclina y la capa de mínimo oxígeno, que limitaron su distribución al estrato superficial (0-50 m), más cálido y más oxigenado. 


\section{AGRADECIMIENTOS}

Los autores agradecen la valiosa colaboración del Dr. Francesc Pagès y Stefanía Navarro en la identificación de algunas especies de medusas. Además, agradecen el financiamiento otorgado por CONICYT a través del Proyecto FONDECYT 1000419, que permitió el desarrollo de esta investigación.

\section{REFERENCIAS}

Alvariño, A. 1985. Predation in the plankton realm, mainly with reference to fish larvae. Invest. Mar. Cicimar, 2: 1-122.

Escribano, R. \& P. Hidalgo. 2000. Spatial distribution of copepods in the north of the Humboldt Current region off Chile during coastal upwelling. J. Mar. Biol. Ass. U.K., 80: 283-290.

Escribano, R. \& I.A. McLaren. 1999. Production of Calanus chilensis in the upwelling area of Antofagasta, northern Chile. Mar. Ecol. Prog. Ser., 177: 147-156.

Escribano, R., V. Marín \& P. Hidalgo. 2001. The influence of coastal upwelling on the distribution of Calanus chilensis in the Mejillones Peninsula (northern Chile): implications for its population dynamics. Hidrobiologia, 453/454: 143-151.

Escribano, R., V. Marín \& C. Iribarren. 2000. Distribution of Euphausia mucronata at the upwelling area of Peninsula Mejillones, northern Chile: the influence of the oxygen minimum layer. Sci. Mar., 64(1): 69-77.

Escribano, R., V. Marín, P. Hidalgo \& G. Olivares. 2002. Physical-biological interactions in the pelagic ecosystem of the nearshore zone of the northern Humboldt Current System. En: J.C. Castilla \& J. Largier (eds.). The oceanography and ecology of the nearshore and bays in Chile. Ediciones Universidad Católica de Chile, Santiago, pp. 145-175.

Fancett, M.S. \& G.P. Jenkins. 1988. Predatory impact of Scyphomedusae on ichthyoplankton and other zooplankton in Port Phillip Bay. J. Exp. Mar. Biol. Ecol., 116: 63-77.

Fagetti, E. 1973. Medusas de aguas chilenas. Rev. Biol. Mar., Valparaíso, 15(1): 31-75.
Gasca, R. 1996. Especies y abundancia de sifonóforos (Cnidaria: Hydrozoa) en la región sur del golfo de México. Carib. J. Sci., 29: 220-225.

Gili, J.M., F. Pagès \& F. Vives. 1987. Distribution and ecology of a population of planktonic cnidarians in the western Mediterranean. En: J. Bouillon, F. Boero \& P. Cornelius (eds.). Modern trends in the systematics, ecology, and evolution of hydroids and hydromedusae. Oxford University Press, Oxford, pp. 157-170.

Gili, J.M., F. Pagès, A. Sabatés \& J.D. Ros. 1988. Small-scale distribution of a cnidarian population in the western Mediterranean. J. Plankton Res., 10(3): 385-401.

Gili, J.M., F. Pagès \& X. Fusté. 1991. Mesoscale coupling between spatial distribution of planktonic cnidarians and hydrographic features along the Galician coast (Northwestern Iberian Peninsula). Sci. Mar., 55(2): 419-426.

González, H., G. Daneri, D. Figueroa, J.L. Iriarte, N. Lefevre, G. Pizarro, R. Quiñones, M. Sobarzo \& A. Troncoso. 1998. Producción primaria y su destino en la trama trófica pelágica y océano profundo e intercambio océano-atmósfera de $\mathrm{CO}_{2}$ en la zona norte de la Corriente de Humboldt $\left(23^{\circ} \mathrm{S}\right)$ : posibles efectos del evento El Niño, 1997-98 en Chile. Rev. Chil. Hist. Nat., 71: 429-458.

Graham, W., J. Field \& D. Potts. 1992. Persistent "upwelling shadows" and their influence on zooplankton distributions. Mar. Biol., 114: 561570 .

Hutchings, L., G.C. Pitcher, T.A. Probyn \& G.W. Bailey. 1995. The chemical and biological consequences of coastal upwelling. En: C.P. Summerhayes, K.-C. Emeis, M.V. Angel, R.L. Smith \& B. Zeitzschel (eds.). Upwelling in the ocean: modern processes and ancient records. John Wiley \& Sons, Chichester, pp. 65-81.

Madin, L.P. \& G.R. Harbison. 1978. Thalassocalyce inconstans, new genus and species, an enigmatic ctenophore representing a new family and order. Bull. Mar. Sci., 28(4): 680-687.

Marazzo, A. \& C. Nogueira. 1996. Composition, spatial and temporal variations of Chaetognatha in Guanabara bay, Brazil. J. Plankton Res., 18(12): 2367-2376. 
Marín, V., L. Delgado \& R. Escribano. 2003. Upwelling shadows at Mejillones bay (northern Chilean coast): a remote sensing in situ analysis. Invest. Mar., Valparaíso, 31(2): 47-55.

Marín, V., L. Rodríguez, L. Vallejo, J. Fuenteseca \& E. Oyarce. 1993. Efectos de la surgencia costera sobre la productividad primaria primaveral de bahía Mejillones del sur (Antofagasta, Chile). Rev. Chil. Hist. Nat., 66: 479-491.

Matsakis, S. \& R. Conover. 1991. Abundance and feeding of Medusae and their potential impact as predators on other zooplankton in Bedford Basin (Nova Scotia, Canada) during Spring. Can. J. Fish. Aquat. Sci., 48: 1419-1430.

Maturana, C. 1999. Relación espacial entre hidromedusas dominantes y los estadios tempranos de desarrollo de Engraulis ringens (Jenyns, 1842) y Strangomera bentincki (Norman, 1936), durante el desove de invierno de 1996, en el área centro sur de Chile. Tesis de Biología Marina, Universidad Arturo Prat, Iquique, 75 pp.

Morales, C., J. Blanco, M. Braun, H. Reyes \& N. Silva. 1996. Chlorophyll- $a$ distribution and associated oceanographic conditions in the upwelling region off northern Chile during the winter and spring 1993. Deep-Sea Res., 43: 267289.

Olivares, G. 2000. Mecanismos de interacción físicobiológica en una zona de surgencia costera, retención de larvas y cierre del ciclo de vida de Euphausia mucronata. Tesis para optar al grado de Magíster en Ciencias Biológicas mención Ecología, Universidad de Chile, 50 pp.

Pagès, F., H. González, M. Ramón, M. Sobarzo \& J.M. Gili. 2001. Gelatinous zooplankton assemblages associated with water masses in the Humboldt Current System, and potential predatory impact by Bassia bassensis (Siphonophora: Calycophorae). Mar. Ecol. Progr. Ser., 210: 13-24.

Pagès, F. \& J.M. Gili. 1992. Influence of Agulhas waters on a population structure planktonic cnidarians in the southern Benguela Region. En: J. Bouillon, F. Boero, F. Cicogna, J-M. Gili \& R.G. Hughes (eds.). Sci. Mar., 56(2-3): 109-123.

Palma, S. 1977. Contribución al estudio de los sifonóforos encontrados frente a la costa de Valparaíso. Aspectos ecológicos. Memorias Segundo
Simposio Latinoamericano de Oceanografía Biológica, Cumaná, 2: 119-133.

Palma, S. 1994. Distribución del macroplancton gelatinoso en un área de desove de peces frente a la costa central de Chile $\left(32^{\circ}-33^{\circ} \mathrm{S}\right)$. Rev. Biol. Mar., Valparaíso, 29(1): 23-45.

Palma, S. \& S. Rosales. 1995. Composición, distribución y abundancia estacional del macroplancton de la bahía de Valparaíso. Invest. Mar., Valparaíso, 23: 49-66.

Purcell, J.E. 1985. Predation on fish eggs and larvae by pelagic cnidarians and ctenophores. Bull. Mar. Sci., 37(2): 739-755.

Purcell, J.E. 1991. A review of cnidarians and ctenophores feeding on competitors in the plankton. Hydrobiologia, 216/217: 335-342.

Purcell, J.E. 1997. Pelagic cnidarians and ctenophores as predators: selective predation, feeding rates, and effects on prey populations. Ann. Inst. Océanogr., Paris, 73(2): 125-137.

Rodríguez L., O. Zárate \& E. Oyarce. 1986. Producción primaria del fitoplancton y su relación con la temperatura, oxígeno, nutrientes y salinidad en la bahía de Mejillones del Sur. Rev. Biol. Mar., 22(1): 75-96.

Rodríguez, L., V. Marín, M. Farías \& E. Oyarce. 1991. Identification of an upwelling zone by remote sensing and in situ measurements, Mejillones del Sur Bay (Antofagasta-Chile). Sci. Mar., 55: 467473.

Santibáñez, P. 2003. Distribución y abundancia de zooplancton quitinoso en relación a condiciones hidrográficas estacionales frente a la bahía de Mejillones (23ㅇ), Chile. Tesis de Biología Marina, Escuela de Biología Marina, Universidad Austral de Chile.

Sievers, H. \& N. Silva. 1982. Masas de agua y circulación geostrófica frente a la costa de Chile, latitudes $18^{\circ} \mathrm{S}$ y $33^{\circ} \mathrm{S}$. Cienc. Tecnol. Mar., 6: 61-99.

Strub, T., J. Mesías, V. Montecino, J. Rutllant \& S. Salinas. 1998. Coastal ocean circulation off western south America. Coastal segment (6,E). En: A. Robinson \& K. Brink (eds.). The Global Coastal Ocean. First Workshop on Coastal Ocean Advanced Science and Technology Studies. IOC, John Wiley \& Sons, Liege. The Sea, 11: 273-313. 
Ulloa, R., S. Palma, L. Linacre \& N. Silva. 2000. Seasonal changes in the bathymetric distribution of siphonophores, chaetognaths and euphausiids associated to water masses off Valparaíso, Chile
(Southeast Pacific). En: J. Farber (ed.). Oceanography Eastern Pacific. CICESE, Ensenada, 1: 7283.

Recibido: 23 mayo 2003; Aceptado: 26 marzo 2004 